\title{
Antibody Fab Fragments and their Crystal Organization
}

\author{
D.T. Gallagher, I. Kargeorgos, and C.V. Galvin
}

\section{NIST/IBBR}

Medical research continues to expand its use of antibodies for the development of new and specifically targeted molecular strategies. This research has produced a wide array of crystal structures including antibodies, their fragments, and complexes. Here we survey this structural family, focusing on Fabs, and examine in detail the crystal packing in the structure $5 \mathrm{~K} 8 \mathrm{~A}$ of the Fab fragment of the NIST reference antbody RM8671. This well refined 2.0 A structure provides a basis for comparisons with relevant precedents and structural neighbors. Comparisons reveal several themes and recurring patterns, especially in the contact-forming surface regions. These patterns, along with crystal conditions data, carry implications for mechanisms of crystallization, antibody aggregation and the development of further antibody engineering strategies. 